\title{
Coordination Structures and Mechanisms for Crisis Management in \\ China: Challenges of Complexity
}

Tom Christensen

Professor of Public Administration and Policy

Department of Political Science, University of Oslo, Norway

Visiting Professor

School of Public Administration and Policy, Renmin University of China, China

Email: tom.christensen@stv.uio.no

Liang Ma

Associate Professor of Public Administration

School of Public Administration and Policy, Renmin University of China, China

Email: liangma@ruc.edu.cn

Tel: 86106251 1122; Fax: 861062516240

Acknowledgement: An earlier version of this manuscript was presented at the Conference "From the management of crisis to the governance of risk: Time for a paradigm shift?", Haikou, Hainan, China, January 9-11, 2017. The authors would like to thank Yana Lu, Yanwei Li, and other conference participants for helpful comments. 


\begin{abstract}
:
When responding to crises, a joint approach is often used, which requires coordination among government agencies and other institutions. In this article we combine the vertical and horizontal dimensions to develop a theoretical framework to explain the patterns and performance of coordination. By drawing on structural-instrumental and institutional perspectives, we examine the coordination structures and mechanisms in China's crisis management at the central and local levels. We outline the main structural characteristics of the Chinese system and the overall challenges it faces. We then discuss four crises that have happened in and around Beijing, using these to understand the crucial factors driving coordination successes and failures. Lastly, we discuss some comparative features and the theoretical and policy implications of our main results.
\end{abstract}

Keywords: Coordination, structure, crisis management, risk, emergency management, China 


\section{Introduction}

The world is characterized by ubiquitous risks and frequent discontinuities posed by crises, whether natural disasters or man-made emergencies, which is attracting increasing attention from political executives and use of resources. Crises are typically "wicked problems" with a high level of uncertainty, unpredictability, complexity and intertwinement, straddling different levels, sectors and organizations (Weber \& Khademian, 2008). In marked contrast to normal, routine operations, the response to crises often requires the orchestrated coordination of multiple government agencies and other institutions. Crisis management is first and foremost a coordinated process (Boin \& 't Hart, 2003), requiring government agencies and various entities to work together to prepare for, handle and recover from crises (Boin \& Lodge, 2016). In other words, coordination among government agencies and social entities during crises is pivotal to governance capacity and crisis management performance, but pose a lot of challenges in practice (Christensen et al., 2016; Hammond, 2007). But governance capacity interacts in crucial ways with governance legitimacy. Coordinated action may be either enhanced or undermined by the extent to which the cultural paths of different public organizations coincide; or by horizontal societal accountability processes whereby citizens' support of or trust in crisis management is crucial.

Coordination is particularly crucial in giant, sprawling countries such as China (Chen, 2016), which faces major challenges in coordinating numerous actors with distinct values and interests (Lu \& Xue, 2016) and still has only a nascent understanding of the coordination structures and mechanisms required for crisis management (Guo \& Kapucu, 2015). China's government has been very keen to learn from the West, particularly in adopting various reform measures 
advocated by the New Public Management (NPM) bandwagon (Chou 2009). The fragmented bureaucratic structures produced by NPM reforms have, however, significantly heightened the coordination challenges of crisis management. But "similar to many Western countries, China is encountering the problem of balancing the structural design of centralization and coordination with the autonomy of lower levels of government and other institutions" (Christensen \& Fan, 2016, p. 12). To address the negative and unintended ramifications of NPM reforms, the government in China has introduced various post-NPM reforms. For instance, the government implemented two rounds of restructuring reforms in 2008 and 2013 to streamline interagency coordination by merging multiple ministries into so-called "superministries" (Dong et al., 2010).

Our research questions in this study are as follows:

- What are the general characteristics of coordination in China's crisis management system and what are some of its potential challenges?

- What are some of the key factors leading to coordination successes and failures in preventing and handling crises? How can we illustrate this with a set of cases in and around Beijing?

- How can we interpret and explain the coordination successes and failures in crisis responses using a set of organization theory perspectives?

In this study, we draw on structural-instrumental and institutional perspectives to examine coordination structures and mechanisms in China's crisis management system, using archival data and interview material to support our arguments. We identify the key characteristics and challenges of coordination in China's crisis management at the national and local levels and use these to discuss the possible future development, implications and comparative perspectives of crisis management in China. 
The article is structured as follows. We first define our key concepts and briefly introduce the frameworks and theories used in this study. We then summarize the key components and instruments of national coordination in China's crisis management and identify crucial mechanisms underpinning coordination in crisis responses and the challenges they pose. We then discuss four crisis management cases in and around Beijing, followed by a more general discussion of China's crisis management from the perspectives mentioned above. We conclude by considering the implications for crisis management reforms and future research avenues.

\section{Concepts and theories}

\section{Coordination structures and mechanisms}

Coordination is about the adjustment of actions and decisions among interdependent actors to achieve a specific goal, which can be done either ex ante or ex post (Christensen et al., 2016, p. 893). Organizations are structured according to the principle of division of labor to maximize work efficiency, which usually is at odds with the need to coordinate cross boundaries within and between organizations (Gulick, 1937). The assets, routines, roles and skills developed for specialization may, however, hinder coordination and cooperation across distinct organizations or units.

Coordination among government agencies and social entities (e.g., NGOs) during crises is pivotal to governance capacity and crisis management performance. The nature of tasks matters, as do scale or scope, because it largely determines the needs and conditions of coordination per se. Crisis management is characterized by uncertainty, instability, complexity, and ambiguity, which requires forms of coordination rather different to those used for stable and routine 
situations and tasks (Schneider, 1992). For "wicked problems" crises and/or disasters, coordination between actors and organizations with differing tasks and perceptions is crucial (Christensen et al., 2016, p. 892). Without strong coordination capacity to mobilize various entities to respond concertedly to emergencies, crisis management can neither succeed nor be sustained. As one of four types of governance capacity (the others are analytical, regulatory, and delivery capacity), coordination capacity entails "bringing together disparate organizations to engage in joint action".

In an increasingly multi-sectoral and multilevel system, actors work across sectors and levels, and coordination can be distinguished in multiple dimensions. Coordination in crisis management can be in various modes and forms, but we can classify it into primarily two umbrella approaches (Christensen et al., 2016, p. 888). The first one is a top-down hierarchical approach, which we focus on in this study. The second is a bottom-up network approach emerging from negotiation, voluntary agreement, and consensus-building (Moynihan, 2009). Government agencies, for instance, have to coordinate with private and nonprofit organizations to respond to crises (Comfort \& Kapucu, 2006). Apart from the two pure forms of coordination, there are also different combinations of the two, which result in hybrid approaches (Kapucu, 2006).

Coordination is not only about formal structures and procedures, but also pertinent to informal values and cultural norms. "[G]overnance capacity includes formal structural and procedural features of the governmental administrative apparatus but also informal elements, that is, how these features work in practice" (Christensen et al., 2016, p. 888). It is quite common that institutions involved in response plans are unable to work together when facing 
crises (Burby, 2005). It is thus important to pay attention to the congruence between coordination plans on paper and how they work in practice. The gap between stipulated and practiced coordination in crisis management merits further theoretical considerations.

The extent to which coordination is actively used and needed in crisis management will vary according to type of political-administrative structures and cultures, situations and type of crisis. Even though we focuses of the use of different types of coordination in crisis management in practice in China, we acknowledge the view that in crisis management the acute phase of handling may be characterized by lack of central coordination and locally based actions (Scholtens, 2008).

McConnell (2011:68) discusses the different dimensions of how we can understand the success or failure of crisis management:

A crisis management initiative is successful if it follows pre-anticipated and/or relevant processes and involves the taking of decisions which have the effect of minimizing loss of life/damage, restoring order and achieving political goals, while attracting universal or near universal support and/no or virtually no opposition.

This definition contains the three dimensions of process, decisions and politics, all of which contain objective elements and perceptions/subjectivity (McConnell, 2011). He also contains that it's rather seldom that success and failure is clear-cut and that there is a spectrum also containing values like durable success (success outweigh failures), conflicted success (success and failure fairly equally balanced) and precarious success (failure outweigh success) (McConnell, 2011, p. 71). 
As a set of rules and roles specifying "who is expected to do what, and how", organizational structure "broadly defines the interests and goals that are to be pursued and the considerations and alternatives that should be treated as relevant" (Egeberg, 2012, p. 158). Structure can be examined from two dimensions, namely vertical and horizontal specialization and coordination (Gulick, 1937). Horizontal specialization is about how different issues and policy areas are supposed to be linked together or de-coupled from each other. Vertical specialization, on the other hand, refers to "the intended division of labor across hierarchical levels within or between organizations" (Egeberg, 2012, p. 159). Coordination across vertical and horizontal dimensions can be either hierarchical or collegial. In ubiquitous hierarchical structures, decisions are often reached through top-down command. In collegial coordination, in contrast, decisions are usually reached "through arguing, bargaining or voting" among collegial bodies such as committees, task forces, project groups, and so forth. i.e. more network coordination (Egeberg, 2012, p. 159).

By focusing on the vertical-horizontal dimensions of coordination, we can develop a $2 \times 2$ diagram (see Table 1). The strength of coordination on the two dimensions can be either relatively high or low, and their combinations generate four scenarios. Although this dichotomy may simplify the complexities of coordination, it helps us understand the logic of joint actions during and after crises, and connects to type of crisis experienced in China.

Insert Table 1 about here. 
In the upper left quadrant, crisis management is characterized by a high level of vertical coordination but a low level of horizontal coordination. In this case, crises are typically bounded within specific domains or policy areas, and top-down command and bottom-up reporting are the primary coordination approach. Routine, predictable, and localized crises (e.g., mining disasters and air pollution) are coordinated across agencies and localities, as stipulated by disaster-specific response plans. Crisis management tasks can be relatively clearly divided across functions or localities, and agencies or jurisdictions can work in parallel.

In the lower right quadrant, a high level of horizontal coordination is juxtaposed with a low level of vertical coordination. In such a situation, crises are jointly handled by multiple agencies at the same level, while cross-level intervention is relatively weak. This is particularly the case with transboundary crises (e.g., typhoons, flood and river pollution), in which vertical control and steering are often weak and implicit.

In the upper right quadrant, both horizontal and vertical coordination mechanisms are at a high level. In this kind of circumstance, crises may be very unique, uncertain, and usually transboundary (e.g., terrorist attacks, earthquakes, epidemics, and social protests), and the two dimensions of coordination are both indispensable in responding to the crisis. For instance, two or more ministries may simultaneously coordinate a number of local governments, and the two dimensions will work jointly in responding to the crisis.

Lastly, in the lower left quadrant, the strength of coordination in both dimensions is low. Coordination on both dimensions is weak and fragmented, which can be attributed either to weak authorities or to conflicting interests and/or values. Another possibility is that the crisis is confined to a single specific policy area and geographical locality (e.g., fire or food safety), 
which can be addressed without either horizontal or vertical coordination. In this case, crisis management is typically handled in a simple and straightforward way, and coordination is not a priority of crisis management in either dimension. While bottom-up reporting is usually stipulated in response plans, vertical steering by upper-tier authorities is not essential for crisis management.

\section{Theoretical perspectives}

Coordination structures refer to the key actors and institutional arrangements in place for responding to emergencies as well as to the key instruments and processes of crisis management. Coordination in crisis management can be examined from several perspectives, and we draw on structural-instrumental and institutional perspectives (Christensen et al. 2007) to examine coordination structures and mechanisms in China's crisis management system.

The structural-instrumental perspective. It's important to focus on the authority and control of political-administrative leaders, who are supposed to score high on rational calculation, i.e. they know what to do and have insight into how to conduct decision-making processes (Dahl \& Lindblom, 1953). In a hierarchical version of this perspective, top leaders will dominate, while a negotiation version will focus on heterogeneity, different interests and compromises (March \& Olson, 1983). Leaders are primarily supposed to use the design of formal structures to obtain public goals.

Using this perspective on crisis management coordination challenges means focusing on how the formal structure of government on different levels either enhances, modifies or undermines governance capacity and coordination efforts. It also entails looking at what kind 
of structural reforms and changes the leaders use to overcome obstacles and what effect this has. Such an approach will be sensitive to how the different principles of specialization and coordination are used and to the special challenges related to the type of tasks performed, etc.

The cultural-institutional perspective. This perspective emphasizes that culturalinstitutional features or unique identities will develop through mutual adaptation to internal and external pressures (Selznick, 1957). According to this perspective, an institutionalized governance regime is the result of a process of unplanned, gradual, and natural development characterized by path dependence in which informal norms and values play key roles in shaping managerial attitudes and organizational behavior (Christensen et al., 2007).

Using this perspective on crisis management may lead to a focus on continuity, pathdependency and logic of appropriateness, which may either enhance or undermine structuralinstrumental efforts to coordinate (Christensen et al., 2016). Cultural traditions may enhance governance legitimacy or what Easton (1965) labels 'diffuse support'. This could include highlighting the importance of professional norms and values in crisis management. Another line of cultural studies looks at how cultural paths and equilibriums are punctuated, which is highly relevant in the face of a crisis (Baumgartner \& Jones, 1993; Kingdon, 1984). (Streeck \& Thelen, 2005) address whether abrupt change leads to discontinuity or continuity, such as institutional 'back-sliding', or to major system changes.

The symbolic perspective. What matters is how actors like governments present their actions, internally and especially externally, through myths and symbols (Meyer \& Rowan, 1977). This is done by 'window-dressing', i.e. creating favorable images of the organization that are the result of 'double-talk', meaning that leaders talk in one way but eventually decide to act in 
different ways, either because they have no intention of putting what they say into practice or else because they don't know how to act or don't have the resources to (Brunsson, 1989). This 'double' talk may contribute to enhancing the legitimacy of leaders who would like to be seen as rational and modern.

Government legitimacy, namely public perceptions of and attitudes to policies and collective actions, strongly influences crisis management performance, and may either enhance or undermine governance capacity (Christensen et al., 2016). The immediate appearance of top leaders at the scene of a crisis, for instance, is a symbolic yet effective way to retain public legitimacy and trust. Government actions that do not sit well with institutional myths may run counter to coordination. It is thus imperative to pay attention to the symbolic ramifications of government actions in crisis management.

\section{Central coordination structures and overall challenges in China}

China is a large, populous, and diverse country afflicted by many kinds of crises, both natural and man-made (Chen, 2016). Crisis management in China goes back to ancient times, when earthquakes, floods, and famines were a common occurrence (Shi, Xu, \& Wang, 2016). In contemporary China, man-made crises, e.g., accidental explosions, epidemics, and environmental pollution, have become a primary source of casualties. The predominant crisis management approach has been characterized by campaign-style governance, which refers primarily to the temporary political mobilization of government and social resources to address urgent issues (Liu, Lo, Zhan, \& Wang, 2015). When a crisis occurs, the responsible ministries in the disaster-specific domain (e.g., earthquake, civil administration, or police) respond, accompanied by newly established cross-ministry coordinating committees that steer other 
pertinent agencies (Zhong, 2014). The country's centrally planned economy and political totalitarianism mean that the government often proves effective in coordinating agencies and mobilizing resources to respond to and recover from crises (Ma, 2012).

The renewed National Emergency Management System (NEMS) of China is composed of four pillars ('one plan and three sub-systems'): response plans (e.g., master plans, disaster- and sector-specific plans); institutional structures (e.g., emergency management office and interagency committee); response mechanisms (scaling-up and information sharing); and legal framework (i.e., the National Emergency Response Law of 2007) (Lu \& Xue, 2016, p. 415).

At the central level, the Overall National Plan for Responses to Public Emergencies is the Master Plan of national emergency response. This umbrella plan embraces various disasterspecific and sector-specific emergency response plans. The State Council (the cabinet), supported by its standing conference and emergency command centers, is the steering headquarters in crisis management. Established in 2006, the Emergency Management Office (EMO) of the State Council is the primary agency in charge of crisis monitoring, information gathering, and comprehensive coordination in China (see Figure 1).

Insert Figure 1 about here

According to their severity, urgency, and aftermath, emergency events are classified into four grades, namely very severe (I), severe (II), serious (III), and general (IV). Local governments are responsible for serious and general emergencies occurring in their jurisdictions, but uppertier and central governments will intervene to deal with very severe and severe emergencies 
that exceed local governments' resources and capacities.

What are the challenges of the formal crisis management system in China, as described above? How does it work in practice? The Emergency Response Committee and its office (EMO) are the lead agencies of crisis management, with dozens of agencies coordinating responses to sector-specific crises (e.g., natural disasters, accidents, public health, and public security). The roles of these two lines of agencies are ambiguous during and after crises, which hinders horizontal coordination between them and vertical coordination with local governments. Coordination, information sharing and joint actions (either sequential, reciprocal, or simultaneous) is problematic (Hall \& O'Toole, 2000).

The dual, albeit ambiguous and even conflicting roles of the Emergency Management Office (EMO) and emergency command centers jeopardize interagency horizontal coordination and their steering of lower-level governments (Zhong, 2014). In the current emergency response regime, the permanent EMO is responsible for planning and preparing for crises, while the temporary headquarters or disaster-specific command centers are in charge of emergency response. Apart from the Emergency Response Committee (ERC) steering crisis response, there are also a handful of cross-agency coordinating committees responsible for specific disasters (Guo \& Kapucu, 2015). Given their different roles and positions in the regime, their horizontal coordination during crises is quite questionable (Lu \& Xue, 2016).

The differentiating ranking orders of agencies in non-emergency (routine or normal) times do not disappear during emergency crisis management, and may even become amplified. Like in the case of the US Department of Defense coordinating with civil agencies in response to Hurricane Katrina (Moynihan, 2012), agencies' entrenched power and interests further 
complicate the politics of coordination during and after crises.

The authoritarian party-state apparatus is adept in mobilizing the bureaucracy, but its coordination with other social entities is poor. Crisis response is characterized by a high degree of militarization, with ubiquitous use of militarized discourses (e.g., command center) and instruments (e.g., troops). Crisis management is highly politicized, and the government heavily relies on top-down high-stake accountability based on cadre personnel rewards and punishments to give local officials incentives, which is very different from independent and flexible responses in decentralized systems (Ma, 2012). Government actions during crises are also characterized by symbols, and the Party-state apparatus makes intensive use of political propaganda for campaigns and mobilization. Professional involvement is still ad hoc and very limited. Social groups and grassroots organizations play indispensable roles in responding to and recovering from crises, but the government still emphasizes the importance of coordination within its bureaucratic machinery.

Governance legitimacy is equally important for crisis management. Legitimacy refers to "public perceptions, attitudes, and trust vis-à-vis government arrangements for crisis management", which underscores citizens' trust in and support for government input, output, and throughput (Christensen et al., 2016, p. 887). In contrast to Western democracies in which citizens trust local government more than the central authorities, the pattern of citizen trust in government in China is just the opposite. Citizens in China by and large support the one-party regime, but they strongly distrust local governments (Chen, 2017). This hierarchical gap in trust undermines coordination in crisis management, since local governments at the forefront of crisis management are woefully short of governance legitimacy to mobilize the public to 
respond jointly to crises. The state media are strictly controlled by the government for propaganda, whereas online and social media are under arbitrary scrutiny and censorship (King, Pan, \& Roberts, 2013). The lack of transparency and effective communication jeopardizes mutual trust between the state and the society, which hinders the public and communities contributing to coordination during crises (White $\& \mathrm{Fu}, 2012$ ). Citizens affected by crises often have to resort to social media and unofficial channels, which are conducive to the generation and spreading of rumors (Ma, 2008). It is common to witness the public blindly following rumors and relying solely on the grapevine to glean information for disaster rescue, which further aggravates the disorder and chaos that characterize crises (Tong \& Zuo, 2014).

\section{Crisis management at the local level: the case of Beijing}

\section{The crisis management structure in Beijing and the surrounding region}

Given the country's large size and diversity, it is also helpful to examine coordination structures in crisis management at the local level in China. Here we use the case of the capital, Beijing, to analyze local crisis coordination. Local governments at each level mimic the crisis management systems at the central and upper-tier levels. Beijing's crisis management system is similar to that of other jurisdictions (particularly municipalities), but its core position in China's political landscape makes its governance of risk highly rather centralized. The findings reported in this study thus cannot be overgeneralized to other regions. We draw on media reports and case studies of recent crises and responses as well as archival materials pertinent to crisis response.

Beijing's ERC is the steering organ in charge of comprehensive emergency response. Expert and advisory groups are used to provide professional expertise and support. The office of the 
ERC is also the municipal government's general duty office and municipal emergency command center, which directly coordinates emergency preparedness and response. Municipal service centers for emergency and non-emergency alarm support the office of the ERC as first responders, while the field command center is at the forefront of coordinating the emergency response of multiple agencies. District-level emergency command centers, 19 disaster-specific emergency command centers, and temporary emergency command centers are also deployed to jointly respond to crises. A locality-specific and social group-based grassroots emergency response system is helpful in mobilizing the general public and other entities to participate in emergency response.

Insert Figure 2 about here.

The framework of emergency response institutions is a complex interagency network, and both government agencies and nongovernment institutions contribute to coordination in crisis management. According to the Beijing Master Plan for Responses to Emergencies, for instance, pertinent agencies refer to "agencies of the municipal party committee and municipal government concerned with every aspect of emergencies' (e.g., monitoring, preparedness, response, mitigation, and recovery). Crisis response is localized unless scaled up to the central level, and local agencies are the primary responders to emergencies.

Apart from cross-level and cross-function coordination, geographical or regional coordination also has a role to play. There is an obvious tendency evolving from top-down and compulsory coordination to bottom-up and voluntary coordination in China's crisis 
management, particularly in the aftermath of the 2008 Wenchuan earthquake (Zhong, 2014). Given the increasing necessity of cross-boundary, cross-level, and cross-sector coordination, government sectors not only comply with formal institutions of coordination (e.g., joint meeting), but also proactively pursue various forms of voluntary collaboration with others. As part of this, interregional coordination in emergency response has been increasingly emerging in many localities, including the Pan-Pearl River Delta, Yangtze River Delta, and BeijingTianjin-Hebei Metropolitan Region. If we look at the agreements, the majority of coordination is multilateral instead of bilateral (Chen, Suo, \& Ma, 2015). Given its political priority as the capital, Beijing dominates coordination with adjacent provinces and prefectures in crisis management. Beijing is the center of the coordination network, and the neighboring jurisdictions (Tianjin and Hebei) are in the first place expected to contribute to its precedent security and development and then benefit from such involvement if possible. For instance, Beijing has been working closely with adjacent jurisdictions to mitigate deteriorating air pollution since 2011, but policy measures such as compulsory bankruptcy of heavy polluters have served to undermine its economic growth and social stability. When an airpocalypse hits Beijing, the Ministry of Environmental Protection will dispatch dozens of central inspection teams to adjacent provinces to tighten air pollution control. Without strong bargaining power and steering capacity from the central government in this process, however, Beijing cannot guarantee its net benefits from such coordination.

Coordination in crisis management: four cases

We use the theoretical framework in Table 1 and comparative case studies to examine 
coordination challenges in crisis management in China. We selected typical cases in the four scenarios following three considerations. First, the case must be characterized by the coordination pattern in the framework. Second, we chose crises in Beijing and the surrounding region to make them comparable and context-relevant. Third, we paid special attention to crises occurring in the last decade. In case studies of crisis management, it is common to use typical cases because of the occasional nature and incomparability of crises. We use these cases to discuss various combinations of dimensions of the crisis management structure and their implications for coordination practice in crisis management. The data used for case studies are from multiple sources, and we primarily draw on media reports and in-depth research reports to trace the processes and key attributes of the cases. We also interviewed two civil servants working at central and Beijing's Emergency Management Offices to verify the facts and hidden stories behind the scenes of the crises. The interviews are semi-structural and each costed for one and half hours. These empirical materials are mainly used to illuminate the challenges and complexities of coordination in the processes of emergency preparation, responses, and recovery.

The first crisis we focus on is the 719 flood in Hebei province (cases are labeled by the dates of occurrence), which was characterized by a high level of horizontal coordination but a low level of vertical coordination. The flood that engulfed the region on July 19, 2016, hit nearly 10 million residents, claimed more than 250 lives and caused economic losses of over 16 billion RMB Yuan (or 2.7 billion US dollars). The last flood on a similar scale occurred almost 20 years ago (August 1996). Local government and the public were not well prepared for the Hebei flood. Particularly in rural areas of Xingtai and other prefecture-level cities, water 
conservation facilities were not well maintained, and floodwater could not be channeled smoothly through blocked rivers and spillways. The floodwater rushed down at night, and local residents roused from sleep were not alerted by rural grassroots organizations to evacuate in time. The military cooperated with local agencies to rescue local residents, and interagency coordination played a crucial role in mitigating causalities and losses.

The challenges of this crisis were inadequate preparation and prevention rather than handling and rescue (Peng, 2008). Because such incidents occur so seldom, preventive efforts are unlikely to be good, because alertness is low. This is often a vertical coordination problem, meaning that higher level authorities should point to potential dangers and provide resources for lower levels to prepare. And when an incident occurs, in this case the flood, authorities above the lower level must be responsible for alerting citizens through local actors. Lack of preparedness may also relate to local cultural factors, e.g., weak state-society interactions (Chan, 2013).

The second crisis we focus on was the red alert on air pollution in Beijing in December 2015. This was typically coordinated vertically while inter-agency coordination was somewhat weaker. With more than six million vehicles and 20 million residents, Beijing suffers severe smog, particularly during winter time. The municipal government issued a response plan for heavy air pollution in March 2015, which stipulated that the authority would issue blue, yellow, orange, and red alerts once it received forecasts that heavy air pollution would linger for one, two, three, or more than three days respectively. The government issued the first red alert on December 7, followed by the second one on December 18, 2015. By adopting a series of temporary emission control measures, the authorities effectively mitigated atmospheric 
pollution to stop it becoming worse (Xue et al., 2016). The responsibilities of municipal agencies and other institutions are clearly stipulated, and coordination during heavy air pollution is primarily hierarchical and top-down. The Office of Command Center, Propaganda Department, and Information Center jointly released alerts through various media channels. Diverse measures were also taken in the education sector, such as banning outdoor activities for schools and even recommending temporary school closure; by the transportation sector, which severely restricted traffic; and by the economic sector, which halted work at outdoor construction sites and industrial production.

This case demonstrates attempts to implement strong vertical coordination in response to the increasing threat of air pollution. Strong vertical steering also implies rather visible and 'draconian' measures, which may have highly symbolic relevance, so people become aware of the pollution problems. The horizontal challenges of coordination may be explained by cultural factors, because diverse agencies, but not all, do not traditionally see themselves or their tasks as related to pollution. And when strong vertical measures are evident, other agencies may think that someone else will take care of the problems.

The third crisis was the 812 explosion in Tianjin. This one required a high level of coordination on both the vertical and horizontal dimensions. A storehouse of dangerous cargo in Tianjin port of Binhai new district exploded on August 12, 2015, killing 165, leaving 8 missing and 798 injured, and causing 6.87 billion RMB Yuan (or 1.1 billion US dollars) worth of damage. The cargo was owned by a company with political connections, which was hence sheltered by central and local officials. The dangerous cargo had been illegally stored, violating or bypassing workplace safety and other regulations across many agencies, either local or 
central. Tianjin port is jointly managed by Tianjin municipality (e.g., personnel and finance) and the Ministry of Transportation (e.g., investment and operation), while Binhai new district is affiliated with Tianjin municipality. The firefighters from the two jurisdictions sprayed water on the unidentified cargo without first checking whether this was the right thing to do, which triggered a tremendous explosion killing 99 firefighters and leaving 5 missing (Huang \& Zhang, 2015). Given the transboundary complexities and biochemical uncertainty, both the central and local governments became engaged in coordinating the crisis response. The municipal government set up a temporary command center to coordinate rescue and recovery in the field, which was later steered by the minister of public security and the head of State Bureau of Workplace Safety Regulation.

This case is divided according to whether we focus on prevention or handling. Concerning prevention, there seem to be ambiguities in accountability between levels and sectors of authorities, related to both vertical cross-level and horizontal cross-sector coordination, and this was not made better by the authorities protecting a company engaged in illegal practices The handling of the crisis showed increased horizontal and in particular vertical coordination, but failed in one central aspect, which can be seen as partly cultural, namely in the firefighters' lack of knowledge about how to handle hazardous materials.

The fourth crisis concerned the 721 rainstorm in 2012. Here Beijing's crisis coordination was highly problematic along both the vertical and horizontal dimensions. Over 1.9 million residents were hit by unprecedented torrential rain on July 21, which resulted in 79 deaths and economic damage of approximately 11 billion RMB Yuan (or 1.6 billion US dollars). Although the Meteorological Bureau had accurately forecast the arrival of heavy rain well in advance, 
the government response and recovery were poorly organized (Wang, Wang, Wei, \& Ye, 2013). Without sufficient help from the official emergency rescue teams, residents caught in the ensuing flood had to rescue themselves. Given the underperformance of the authority in emergency response, the public was very discontented with the crisis management. For instance, the government launched a campaign to raise online donations, but few people responded and donated. Some citizens even criticized the fund-raising campaign, questioning government legitimacy in eliciting funds from the public. "Although the administration has issued a widespread warning in advance, the city still suffered serious damages and losses from this devastating disaster, which aroused attention as well as criticism from the nation and the world" (Wang et al., 2013, p. 974).

This crisis shows problems of both government capacity and legitimacy. Capacity-wise, the sector expert authority's advice was not handled in an appropriate way, which has both structural and cultural explanations, meaning poor common vertical and horizontal coordination. Added to this, legitimacy and trust were undermined, even though the government used fund-raising as a symbol of support.

\section{Discussion}

Coordination and crisis management performance

The evolution and restructuring processes of the crisis management system in China suggest that it has been transformed into an established regime, albeit with many built-in challenges (Zhang \& Tong, 2016). In responding to key milestones of crises, e.g., the Wenchuan earthquake in 2008, the system performed very well and was successful in responding to 
disasters. In other disasters, like the Sanlu infant milk powder contamination in 2008, however, government responses were poorly organized and learned costly lessons in crisis management. It is intriguing to interpret the celebrated successes and painful failures of coordination in crisis management (Zhang, 2012). Is coordination key to explaining the variations in crisis management performance across the different cases? By examining this question, we can identify the challenges and dilemmas in the coordination of China's crisis management.

Crisis management performance is jointly shaped by governance capacity and governance legitimacy (Christensen et al., 2016). Coordination capacity and its match or mismatch with types of crisis may partially explain government performance in crisis management. In the case of earthquakes and mining disasters, upper-tier entities separately coordinate subordinate entities in parallel pillars. The central government is very strong while local governments are weak, and vertical coordination works effectively in responding to some crises (Zhong, 2014). Particularly by using campaign-style coordination, the central government can successfully provide incentives for its apparatus and mobilize the public in crisis management ( $\mathrm{Lu} \mathrm{\&} \mathrm{Xue,}$ 2016). But the major weakness in the system is the fragmented horizontal structure when it comes to coordination in cross-sectoral and cross-regional crises (Guo \& Kapucu, 2015). The State Council used a pairing-assistance package to assign rich provinces to assist counties and prefectures hit by the 2008 earthquake, which helped these jurisdictions quickly recover from the disaster. In other cases, both dimensions of coordination were fragmented and weak, resulting in chaos and failures in crisis responses.

Concerning overall government legitimacy in the crisis management system, the central government may succeed in creating the feeling that a crisis has been appropriately handled - 
and therefore retain societal support - through the use of diverse symbols, especially using campaign-style efforts. But citizens' reactions, for example through social media, claiming that crises have been badly handled will stick and make it difficult for central authorities to reach the local level with its actions and symbols (Liu \& Chang, 2016). A major challenge concerning government legitimacy is the historical path of mistrust in local government, which makes local actions in crises more difficult.

How can we interpret the four cases based on the three perspectives outlined? First, starting with instrumental factors and government capacity, the cases show that failures, meaning problems of prevention and handling may occur with all combinations of vertical and horizontal coordination, i.e. all four types of scenarios outlined. Overall, preparation and prevention problems are more evident than handling problems (Peng, 2008). There are, however, cases where the handling was relatively good, like the flood and air pollution, or partly successful, like the explosion, so bad preparation did not always lead to bad handling.

Second, government legitimacy, understood as primarily related to cultural and symbolic factors, is in intricate ways connected to government capacity, either strengthening or undermining it (Christensen et al., 2016). Cultural factors played a role in the flood case, for instance, where a weak local culture related to alertness undermined prevention efforts. In the air pollution case sector cultures undermined the handling efforts. In the explosion case, a weak local professional culture aggravated the challenges of local preparation. In the rainstorm case local cultures obstructed both prevention and handling. Symbols seem to have had an impact in both directions. In the pollution case, the leadership managed to use symbols of draconian measures to improve alertness and eventual practice. In the rainstorm case, failed symbols and 
lack of local trust added to problems of horizontal coordination, with respect to both prevention and handling.

Third, coming back to McConnell's (2011) dimensions of success or failure - process, decisions and politics, the process dimension related is mostly a failure in all cases, except the air pollution case, when it comes to prevention. Lack of preparedness was evident, but also spreading information from experts. When it comes to decisions on handling and coordination, it's mostly a success, except for the rainstorm. Politics and legitimacy problems are related to cultural resistance to coordination, with symbolic features.

What can we learn if we compare Chinese crisis coordination with that of other countries? There are both similarities and differences. China has been very proactive in learning from Western countries' practice and experience, also in crisis management (Christensen et al., 2008; Christensen et al., 2012), which makes its coordination structures similar to international ones, e.g., centralized crisis management apparatus, localized administration and scaling-up. Coordination in crisis management in China, however, also differs from that of Western countries in many respects, being more hierarchical, more political, and more militarized. If we compare our main results with a study of coordination in crisis management in six Western European countries, both similarities and differences are evident (Christensen, Danielsen, Lægreid, \& Rykkja, 2016, p. 326). Top administrative leaders in those countries perceived vertical coordination as more important and better than horizontal coordination, which seems to be the same as in China, but it is somewhat weaker in relative terms than it seems to be in China. The administrative coordinative culture in those countries seems, however, to be much more sophisticated than in China, which is unsurprising given the Chinas complex system and 
borne out by our cases.

Our study is limited in three respects. First, government capacity related to vertical and horizontal coordination in crises could have been studied in more detail and in other phases of the crises, but also more explicitly in a longer time perspective. Second, the dynamics related to governance legitimacy and capacity could have been studied more. Our cases show that cultural factors mostly undermine coordination efforts, while symbolic factors both enhance and undermine such efforts. It might be worthwhile to pursue a wider set of cases with more focus on these dynamics. Lastly, our comparison of China with other countries could be expanded in future research. It is intriguing to apply and extend this generic framework to other countries and regions, helping to refine our understanding of coordination in crisis management. For instance, the patterns of coordination would vary in different regimes of crisis management (e.g., networked and leading-agency approaches).

\section{Conclusion}

Coordination lies at the heart of crisis management, which is shown in the challenges of combining centrally controlled crisis management with sector and local coordination. We develop a theoretical framework to combine vertical and horizontal coordination to interpret crisis management performance. The theoretical analysis generates four scenarios, which are illuminated by government responses to four typical crises in Beijing and its adjacent region. We draw on structural-instrumental and institutional perspectives to discuss the main results, and identify the key characteristics and challenges in coordination for crisis management. We also briefly compare the China case with European practices (Christensen, Danielsen, Lægreid and Rykkja, 2016), and identify both similarities and differences. 
The findings contribute to the existing literature on crisis coordination and trust in the following ways: First, they reveal the importance of the dynamics of government capacity and legitimacy in the two dimensions of coordination for effective responses to crises (Christensen et al., 2016). The combined patterns of coordination should match the types of crisis and context attributes (Boin \& Lodge, 2016), otherwise crisis responses cannot succeed or be sustained. Adding to this, lack of trust from the citizens towards the local government or cultural tensions among local sectors or towards central government undermines the effects of coordination.

Second, there is a lot of dynamics and variety between coordination in preparation and handling, and preparation was not any guarantee for good handling. On the other hand, systematic coordination locally was a major precondition for success in local handling of the crises. Third - and this is more of a special point related to the Chinese experience - a fragmented central crisis management apparatus, with ad hoc style political interventions locally, impacted negatively on local coordination efforts.

Even in cases where the government handles a crisis well and implements successful recovery, it is crucial to improve government capacity in prevention and preparedness. The central-local disparity in government legitimacy can jeopardize coordination at the local level, and ineffective communication weakens crisis management capacity (White \& $\mathrm{Fu}, 2012$ ). These findings generate helpful policy implications for the government to strengthen coordination capacity and legitimacy in crisis management. The theoretical framework developed in this study can be used in future studies to compare coordination structures and mechanisms for crisis management across countries, crisis management regimes and cases. 


\section{References}

Baumgartner, F., \& Jones, B. (1993). Agendas and Instability in American Politics. Chicago: University of Chicago Press.

Boin, A., \& 't Hart, P. (2003). Public Leadership in Times of Crisis: Mission Impossible? Public Administration Review, 63(5), 544-553 doi:10.1111/1540-6210.00318

Boin, A., \& Lodge, M. (2016). Designing resilient institutions for transboundary crisis management: A time for public administration. Public Administration, 94(2), 289-298 doi:10.1111/padm.12264

Brunsson, N. (1989). The Organization of Hypocrisy: Talk, Decisions and Actions in Organizations. Chichester: Wiley.

Burby, R. J. (2005). Have State Comprehensive Planning Mandates Reduced Insured Losses from Natural Disasters? Natural Hazards Review, 6(2), 67-81 doi:doi:10.1061/(ASCE)1527-6988(2005)6:2(67)

Chan, H.-y. (2013). Crisis Politics in Authoritarian Regimes: How Crises Catalyse Changes under the State-Society Interactive Framework. Journal of Contingencies and Crisis Management, 21(4), 200-210 doi:10.1111/1468-5973.12024

Chen, B., Suo, L., \& Ma, J. (2015). A Network Approach to Interprovincial Agreements: A Study of Pan Pearl River Delta in China. State and Local Government Review, 47(3), 181-191 doi:10.1177/0160323x15610384

Chen, D. (2017). Local Distrust and Regime Support: Sources and Effects of Political Trust in China. Political Research Quarterly, doi: 1065912917691360 doi:doi:10.1177/1065912917691360

Chen, G. (2016). The Politics of Disaster Management in China: Institutions, Interest Groups, and Social Participation. New York: Palgrave Macmillan.

Christensen, T., Danielsen, O. A., Lægreid, P., \& Rykkja, L. H. (2016). Comparing coordination structures for crisis management in six countries. Public Administration, 94(2), 316332 doi:10.1111/padm.12186

Christensen, T., Dong, L., \& Painter, M. (2008). Administrative reform in China's central government -- how much 'learning from the West'? International Review of Administrative Sciences, 74(3), 351-371 doi:10.1177/0020852308095308

Christensen, T., Dong, L., Painter, M., \& Walker, R. M. (2012). Imitating the West? Evidence on Administrative Reform from the Upper Echelons of Chinese Provincial Government. Public Administration Review, 72(6), 798-806 doi:10.1111/j.1540-6210.2012.02582.x

Christensen, T., \& Fan, Y. (2016). Post-New Public Management: a new administrative paradigm for China? International Review of Administrative Sciences doi:10.1177/0020852316633513

Christensen, T., Lægreid, P., Roness, P. G., \& Røvik, K. A. (2007). Organization Theory and the Public Sector: Instrument, Culture and Myth. Aldershot, UK: Ashgate.

Christensen, T., Lægreid, P., \& Rykkja, L. H. (2016). Organizing for Crisis Management: Building Governance Capacity and Legitimacy. Public Administration Review, 76(6), 887-897 doi:10.1111/puar.12558

Comfort, L. K., \& Kapucu, N. (2006). Inter-organizational coordination in extreme events: The 
World Trade Center attacks, September 11, 2001. Natural Hazards, 39(2), 309-327 doi:10.1007/s11069-006-0030-x

Dahl, R. A., \& Lindblom, C. E. (1953). Politics, Economics, and Welfare. New York: Harper \& Row.

Dong, L., Christensen, T., \& Painter, M. (2010). A Case Study of China's Administrative Reform: The Importation of the Super-Department. The American Review of Public Administration, 40(2), 170-188 doi:10.1177/0275074009334075

Egeberg, M. (2012). How bureaucratic structure matters: An organizational perspective. In B. G. Peters \& J. Pierre (Eds.), The SAGE Handbook of Public Administration (pp. 157168). London: Sage Publications.

Gulick, L. H. (1937). Notes on the Theory of Organization. In L. H. Gulick \& L. F. Urwick (Eds.), Papers on the Science of Administration. New York: Institute of Public Administration.

Guo, X., \& Kapucu, N. (2015). Examining collaborative disaster response in China: network perspectives. Natural Hazards, 79(3), 1773-1789 doi:10.1007/s11069-015-1925-1

Hall, T. E., \& O'Toole, L. J., Jr. (2000). Structures for Policy Implementation: An Analysis of National Legislation, 1965-1966 and 1993-1994. Administration Society, 31(6), 667686 doi:10.1177/00953990022019281

Hammond, T. H. (2007). Why Is the Intelligence Community So Difficult to Redesign? Smart Practices, Conflicting Goals, and the Creation of Purpose-Based Organizations. Governance, 20(3), 401-422 doi:10.1111/j.1468-0491.2007.00364.x

Huang, P., \& Zhang, J. (2015). Facts related to August 12, 2015 explosion accident in Tianjin, China. Process Safety Progress, 34(4), 313-314 doi:10.1002/prs.11789

Kapucu, N. (2006). Interagency Communication Networks During Emergencies: Boundary Spanners in Multiagency Coordination. The American Review of Public Administration, 36(2), 207-225 doi:doi:10.1177/0275074005280605

King, G., Pan, J., \& Roberts, M. E. (2013). How Censorship in China Allows Government Criticism but Silences Collective Expression. American Political Science Review, 107(2), 326-343 doi:doi:10.1017/S0003055413000014

Kingdon, J. W. (1984). Agendas, Alternatives, and Public Policies. Boston: Little, Brown.

Liu, N. N., Lo, C. W.-H., Zhan, X., \& Wang, W. (2015). Campaign-Style Enforcement and Regulatory Compliance. Public Administration Review, 75(1), 85-95 doi:10.1111/puar.12285

Liu, S. N., \& Chang, T.-K. (2016). One Disaster, Three Institutional Responses. Journalism Studies, 1-23 doi:10.1080/1461670X.2016.1190666

Lu, X., \& Xue, L. (2016). Managing the unexpected: Sense-making in the Chinese emergency management system. Public Administration, 94(2), 414-429 doi:10.1111/padm.12261

Ma, C.-C. (2012). Responding in Crises: A Comparative Analysis of Disaster Responses between Mainland China and Taiwan. Journal of Homeland Security and Emergency Management, 9(1), doi: 10.1515/1547-7355.1993 Retrieved from http://www.degruyter.com/view/j/jhsem.2012.9.issue-1/1547-7355.1993/15477355.1993.xml

Ma, R. (2008). Spread of SARS and War-Related Rumors through New Media in China. Communication Quarterly, 56(4), 376-391 doi:10.1080/01463370802448204 
March, J. G., \& Olson, J. P. (1983). Organizing Political Life: What Administrative Reorganization Tells Us about Government. The American Political Science Review, 77(2), 281-296 Retrieved from http://www.jstor.org/stable/1958916

McConnell, A. (2011). Success? Failure? Something in-between? A framework for evaluating crisis management. Policy and Society, 30(2), 63-76 doi:http://dx.doi.org/10.1016/j.polsoc.2011.03.002

Meyer, J. W., \& Rowan, B. (1977). Institutionalized Organizations: Formal Structure as Myth and Ceremony. The American Journal of Sociology, 83(2), 340-363 Retrieved from http://www.jstor.org/stable/2778293

Moynihan, D. P. (2009). The Network Governance of Crisis Response: Case Studies of Incident Command Systems. Journal of Public Administration Research and Theory, 19(4), 895915 doi:10.1093/jopart/mun033

Moynihan, D. P. (2012). A Theory of Culture-Switching: Leadership and Red-Tape During Hurricane Katrina. Public Administration, 90(4), 851-868 doi:10.1111/j.14679299.2011.02017.x

Peng, Z. (2008). Preparing for the Real Storm during the Calm: A Comparison of the Crisis Preparation Strategies for Pandemic Influenza in China and the U.S. Journal of Homeland Security and Emergency Management, 5(1), doi:10.2202/1547-7355.1436 doi:10.2202/1547-7355.1436

Schneider, S. K. (1992). Governmental Response to Disasters: The Conflict between Bureaucratic Procedures and Emergent Norms. Public Administration Review, 52(2), 135-145 doi:10.2307/976467

Scholtens, A. (2008). Controlled Collaboration in Disaster and Crisis Management in the Netherlands, History and Practice of an Overestimated and Underestimated Concept. Journal of Contingencies and Crisis Management, 16(4), 195-207 doi:10.1111/j.14685973.2008.00550.x

Selznick, P. (1957). Leadership in Administration. New York: Harper \& Row.

Shi, P., Xu, W., \& Wang, J. a. (2016). Natural Disaster System in China. In P. Shi (Ed.), Natural Disasters in China (pp. 1-36). Berlin, Heidelberg: Springer Berlin Heidelberg.

Streeck, W., \& Thelen, K. (2005). Institutional Change in Advanced Political Economies. In W. Streeck \& K. Thelen (Eds.), Beyond Continuity: Institutional Change in Advanced Political Economies. Oxford: Oxford University Press.

Tong, J., \& Zuo, L. (2014). Weibo communication and government legitimacy in China: a computer-assisted analysis of Weibo messages on two 'mass incidents' $\uparrow$. Information, Communication \& Society, 17(1), 66-85 doi:10.1080/1369118X.2013.839730

Wang, K., Wang, L., Wei, Y.-M., \& Ye, M. (2013). Beijing storm of July 21, 2012: observations and reflections. Natural Hazards, 67(2), 969-974 doi:10.1007/s11069-013-0601-6

Weber, E. P., \& Khademian, A. M. (2008). Wicked Problems, Knowledge Challenges, and Collaborative Capacity Builders in Network Settings. Public Administration Review, 68(2), 334-349 doi:doi:10.1111/j.1540-6210.2007.00866.x

White, J. D., \& Fu, K.-W. (2012). Who Do You Trust? Comparing People-Centered Communications in Disaster Situations in the United States and China. Journal of Comparative Policy Analysis: Research and Practice, 14(2), 126-142 doi:10.1080/13876988.2012.664688 
Xue, Y., Zhou, Z., Nie, T., Pan, T., Qi, J., Nie, L., . . . Tian, H. (2016). Exploring the Severe Haze in Beijing During December, 2015: Pollution Process and Emissions Variation. Environmental Science, 37(5), 1593-1601

Zhang, H. (2012). What has China Learnt from Disasters? Evolution of the Emergency Management System after SARS, Southern Snowstorm, and Wenchuan Earthquake. Journal of Comparative Policy Analysis: Research and Practice, 14(3), 234-244 doi:10.1080/13876988.2012.687621

Zhang, H., \& Tong, X. (2016). Structural Change in China's Emergency Management: Theoretical Generalizations. Social Sciences in China, 37(2), 77-98 doi:10.1080/02529203.2016.1162010

Zhong, K. (2014). From Obligatory to Voluntary: The Evolution and Development of Emergency Management Coordination Structure in China. Chinese Public Administration(8), 115-119 


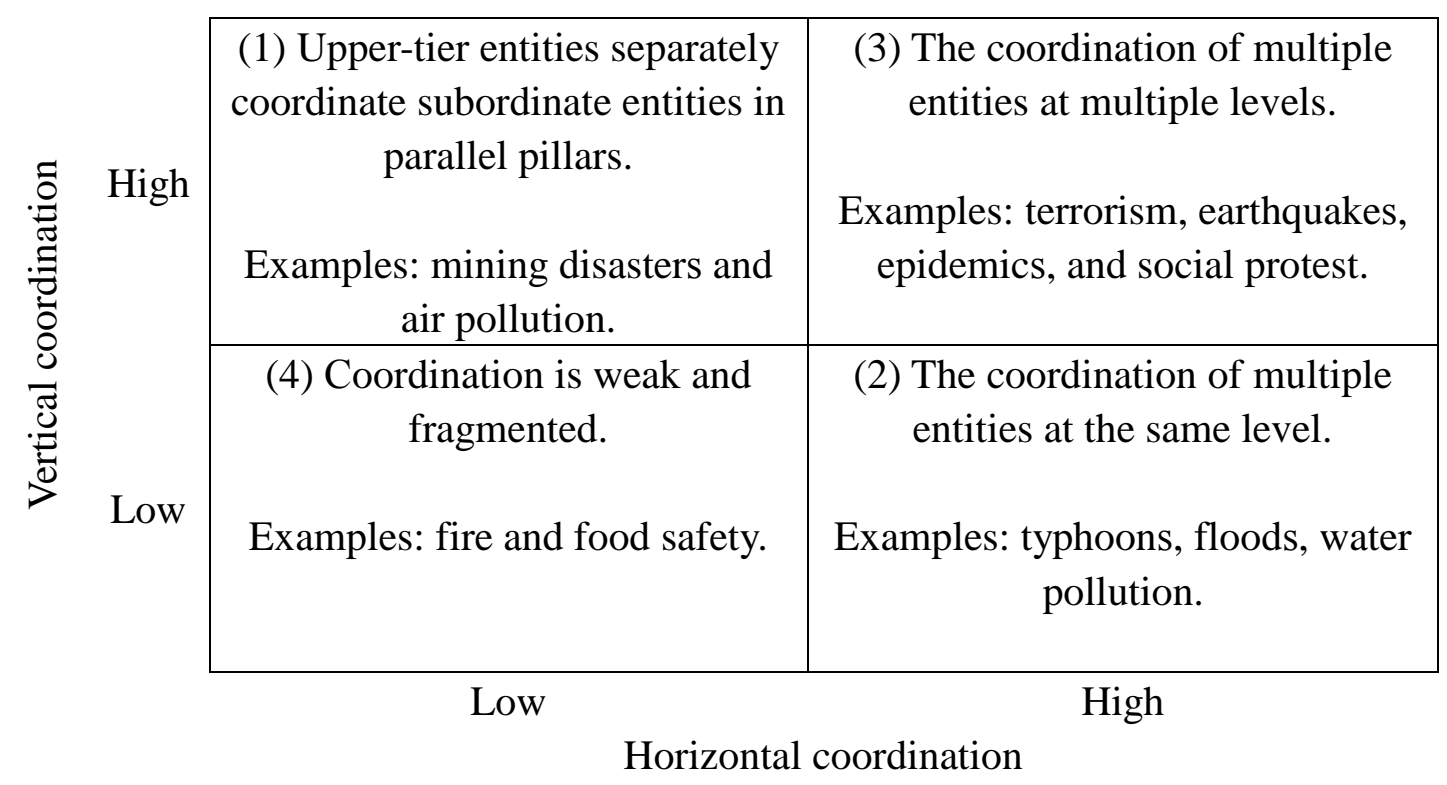

Table 1. Vertical and horizontal coordination in crisis management 


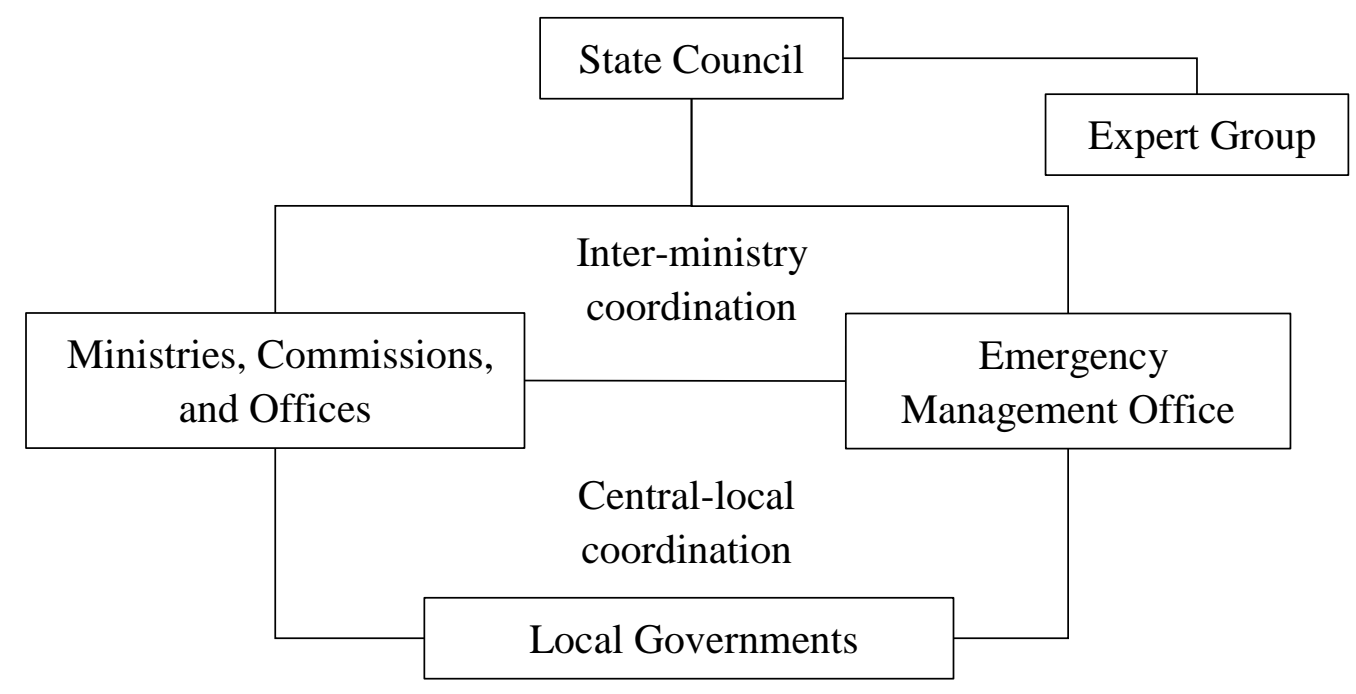

Figure 1. The national crisis management framework in China

Source: http://www.gov.cn/yjgl/2005-08/31/content_69625.htm. 


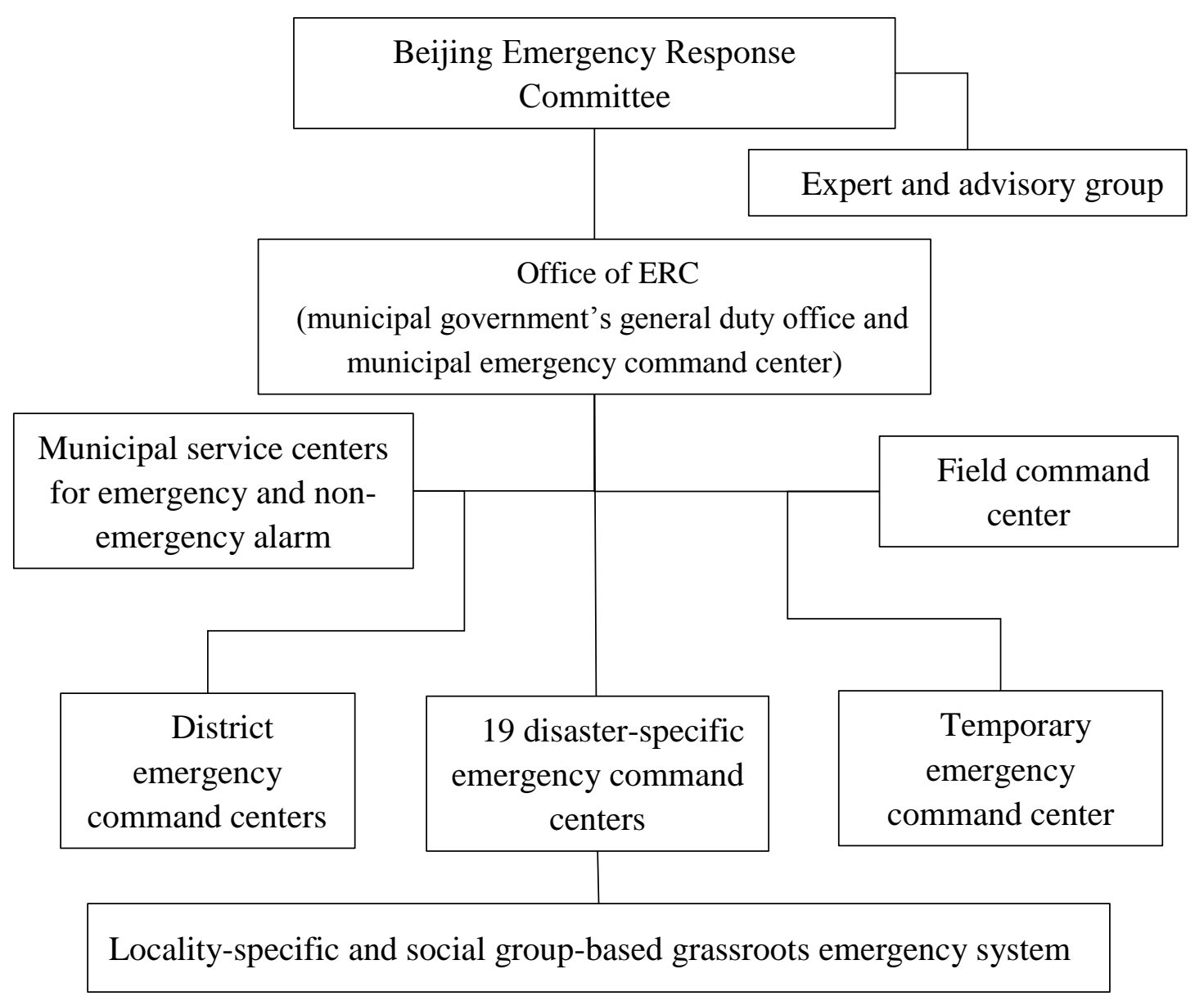

Figure 2. The framework of emergency response institutions in Beijing

Source: http://www.bjyj.gov.cn/yjjg/. 